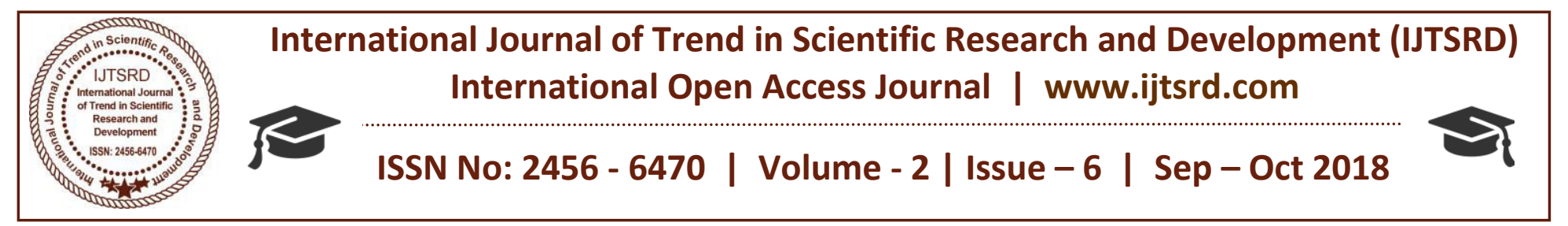

\title{
Single Image Super Resolution using Interpolation \& Discrete Wavelet Transform
}

\author{
Shalini Dubey ${ }^{1}$, Prof. Pankaj Sahu ${ }^{2}$, Prof. Surya Bazal ${ }^{2}$ \\ ${ }^{1}$ Research Scholar, ${ }^{2}$ Assistant Professor \\ Department of Electronics and Communication Engineering, \\ Gyan Ganga Institute of Technology and Sciences, \\ Jabalpur, Madhya Pradesh, India
}

\section{ABSTRACT}

An interpolation-based method, such as bilinear, bicubic, or nearest neighbor interpolation, is regarded as a simple way to increase the spatial resolution for the LR image. It uses the interpolation kernel to predict the missing pixel values, which fails to approximate the underlying image structure and leads to some blurred edges. In this work a super resolution technique based on Sparse characteristics of wavelet transform. Hence, we proposed a wavelet based super-resolution technique, which will be of the category of interpolative methods, using sparse property of wavelets. It is based on sparse representation property of the wavelets. Simulation results prove that the proposed wavelet based interpolation method outperforms all other existing methods for single image super resolution. The proposed method has $7.7 \mathrm{~dB}$ improvement in PSNR compared with Adaptive sparse representation and self-learning ASR-SL [1] for test image Leaves, 12.92 $\mathrm{dB}$ improvement for test image Mountain Lion \& 7.15 $\mathrm{dB}$ improvement for test image Hat compared with ASR-SL [1]. Similarly, 12\% improvement in SSIM for test image Leaves compared with [1], 29\% improvement in SSIM for test image Mountain Lion compared with [1] \& 17\% improvement in SSIM for test image Hat compared with [1].

Keyword: Super Resolution, Image Reconstruction, Single Image Resolution Techniques, Resolution Enhancement, Wavelet transform, Interpolation.

\section{INTRODUCTION}

Image super resolution is an image processing algorithms that produce high quality, high resolution (HR) images from a set of low quality, low resolution
(LR) images or from a single image. The SR image reconstruction is useful in many practical cases where multiple frames of the same scene can be obtained; including medical imaging, satellite imaging, and video applications [17].The basic premise for increasing the spatial resolution in SR techniques is the availability of multiple LR images captured from the same scene. The set of source low resolution (LR) images captures only a finite amount of information from a scene; the goal of SR is to extract the independent information from each image in that set and combine the information into a single high resolution (HR) image. The requirement is of $S R$ is that each LR image must contain some information that is unique to that image [3]. The super resolution method is to take more samples of the scene so as to get some extra information which can be used, while merging the samples to get a high resolution image. These samples can be acquired by sub-pixel shifts, by changing scene , by changing the amount of blur [14] .HR means that pixel density within an image is high, and therefore an HR image can offer more details that are important in many applications, The major advantage of the super resolution approach is that it may cost less and the existing LR imaging systems can be still utilized. Synthetic zooming of region of interest (ROI) is another important application in surveillance, forensic, scientific, medical, and satellite imaging [13]. This application is most suitable for magnifying objects in the scene such as the face of a criminal or the license plate of a car [16]. 


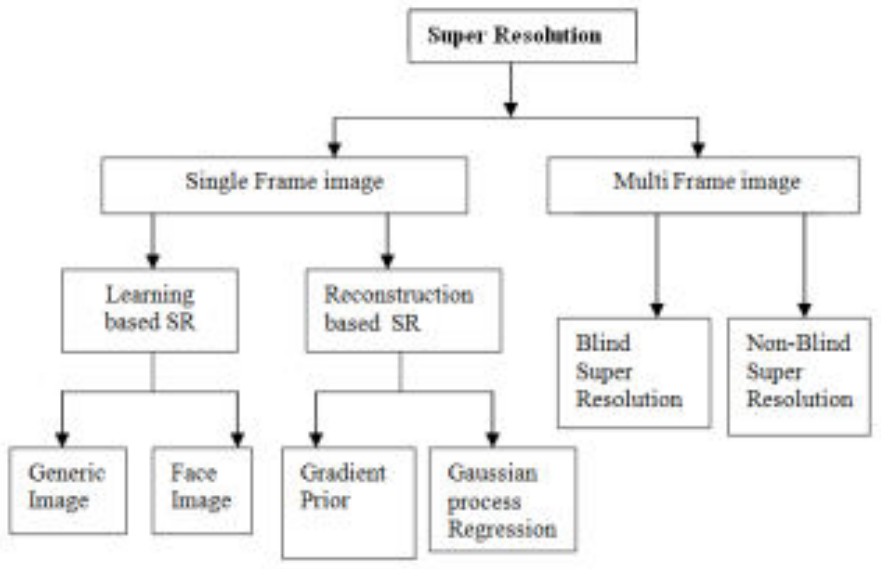

Figure 1: Classification of Super Resolution Techniques

\section{VARIOUS EXISTING SUPER- RESOLUTION TECHNIQUES}

2.1 SR using compressive sensing based on a redundant dictionary

1. Y. Sun Et-al, presented a compressive sensing based on a redundant dictionary has been successfully applied in super resolution imaging. However, due to the neglect of the local and nonlocal interactions of patches of a single image, the reconstructed results are not satisfactory in noise suppression and edge sharpness. In this paper, we propose an improved method by adding steering kernel regression and a nonlocal means filter as two regularization terms and use an efficient clustering sub-dictionary learning scheme. We further demonstrate better results on true images in terms of traditional image quality assessment metrics.

\subsection{SR reconstruction based on the compressive} sensing

2. Y. Sun Et-al, presented an observation for medical imaging and astronomical, high-resolution (HR) images are urgently desired and required. In recent years, many researchers have proposed various ways to achieve the goal of image super-resolution (SR), ranging from simple linear interpolation schemes to nonlinear complex methods. In this paper, we deal with the SR reconstruction problem based on the theory of compressive sensing, which uses a redundant dictionary instead of a conventional orthogonal basis. We further demonstrate better results on true images in terms of peak signal-to-noise ratio (PSNR) and root mean-square error (RMSE) and give several important improvements, compared with other methods.
Although wide variety of super-resolution literature is available, it is still an open topic to investigate. Following subsections describe some of the existing basic image super-resolution schemes.

\subsection{SR via Sparse Representation}

Jianchao Yang et al. [9] considered the sparse signal representation of an image. Based on previous research on image statistics the image patches can be well-represented as a sparse linear combination of elements from an appropriately chosen over-complete dictionary. Motivated by this, they proposed a sparse representation for each patch of the low-resolution input. The coefficients of this representation are used to generate the high-resolution output. Theoretical results from compressed sensing suggest that the sparse representation can be correctly recovered from the down-sampled signals under mild conditions.

Similarity of sparse representations between the low-resolution and high-resolution image patch pair with respect to their own dictionaries is enforced, by jointly training two dictionaries for the low and highresolution image patches. So, the sparse representation of a low-resolution image patch is being applied with the high-resolution image patch dictionary to generate a high-resolution image patch. They showed the effectiveness of such a sparsity prior for both general image super-resolution (SR) and the special case of face hallucination. This algorithm can handle SR with noisy inputs in a more unified framework because the local sparse modeling is naturally robust to noise.

\subsection{Nonlinear Mapping of Coherent Features}

Xiao Zeng and Hua Huang presented a regression based method that can successfully recognize the identity given all these difficulties. They built a radial basis function in subspace by canonical correlation analysis to nonlinear regression models from the specific non frontal low resolution image to frontal high resolution features.

\subsection{Geometric Grouplets}

A. Maalouf and M. C. Larabi proposed the idea of generating a super-resolution (SR) image from a single multi-valued low-resolution (LR) input image. This problem approaches from the perspective of image geometry-oriented interpolation. They computed the group let transform to obtain geometry of the LR image. Geometric group lets is constructed 
by orthogonal multiscale grouping with weighted Haar lifting to points grouped by association fields.

To preserve the sharpness of edges and textures SR image is synthesised by an adaptive directional interpolation using the extracted geometric information. This method showed improvements over existing geometrically driven interpolation techniques on a subjective scale, and in many cases with an improvement in psycho visual color difference.

\subsection{Remotely Sensed image by Hope field Neural Network}

J Tatem Andrew et al. [12] used their idea of superresolution for target identification in remotely sensed images. Fuzzy classification improves the accuracy of land cover target identification make robust and better for spatial representation of land cover. The Hopfield neural network converges to a minimum of an energy function, defined as a goal and several constraints. The energy minimum represents a best guess map of the spatial distribution of class components in each pixel.

They used two goal functions to make the output of a neuron similar to that of its neighboring neurons. The first goal function aims to increase the output of center neuron to 1 . The second goal function aims to decrease the output of the center neuron to 0 . They showed that, by using a Hopfield neural network, more accurate measures of land cover targets can be obtained compared with those determined using the proportion images alone.

\subsection{Neural Network based Optimal Recovery Theory}

Yizhen Huang and YangjingLong [13] proposed Motivated by the idea that back propagation neural network are capable of learning complex nonlinear function they proposed a neural network approach that produces better results in high-frequency regions. They integrated an optimal recovery based approach with in a neural network framework and, if so, two different branches of algorithms complement each other to offer a better algorithm. Using this algorithm in a two-pass way generates visual results that are very similar regardless of the initial interpolation step, and more times of iteration only waste the computing resource but yield negligible performance gain.

\subsection{Gaussian Process Regression}

Wan-Chi Siu et-al, [14] addressed the problem of producing a high-resolution image from a single low- resolution image without any external training set. They proposed a framework for both magnification and deblurring using only the original low-resolution image and its blurred version. In This method, each pixel is predicted by its neighbors through the Gaussian process regression.

They showed that, by using a proper covariance function, the Gaussian process regression can perform soft clustering of pixels based on their local structures. This algorithm can extract adequate information contained in a single low-resolution image to generate a high-resolution image with sharp edges. Compared to other edge-directed and examplebased super-resolution algorithms this algorithm is superior in quality and performance.

\section{9 // Learning-based SR with a combining of both global and local constraints}

K. Guoet al. [15] proposed a statistical learning method for SR with both global and local constraints. More specifically, they introduced a mixture model into maximum a posteriori (MAP) estimation, which combines a global parametric constraint with a patchbased local non-parametric constraint.

The global parametric constraint guarantees the superresolved global image to agree with the sparse property of natural images, and the local nonparametric constraint is used to infer the residues between the image derived from the global constraint and the ground truth high-resolution (HR) image.

They compared it with traditional patch-based learning methods without the global constraint, and

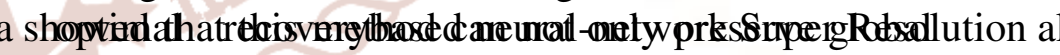
image structure, but also restore the local details more effectively.

\subsection{Interpolation based SR using Multi surface Fitting}

Fei Zhou et al. [16] proposed a interpolation-based method of image super-resolution reconstruction. They used the idea of multi surface fitting to take advantage of spatial structure information. Each site of low-resolution pixels is fitted with one surface, and the final estimation is made by fusing the multisampling values on these surfaces in the maximum a posteriori fashion. Figure 2 shows the flow chart of interpolation based SR using Multi surface Fitting. 


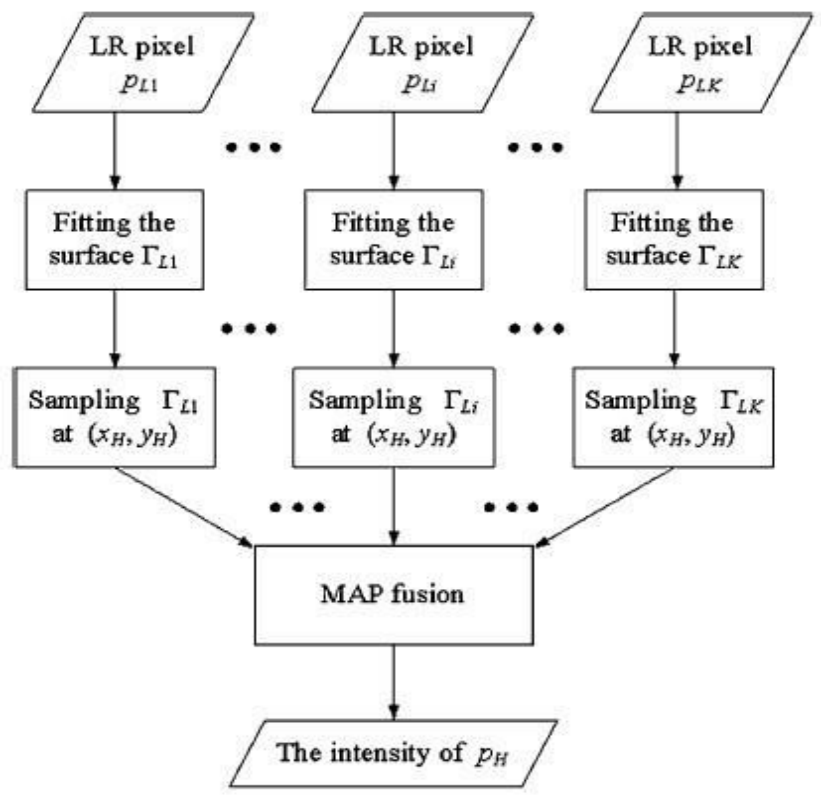

Figure 2: Flow chart of Multi surface Fitting

For the final values of high intensity pixels they used maximum a posteriori estimation on sampled surface constructed using Taylor series. They showed that, this method reconstructs high-resolution images that preserve image details effectively without any hypothesis on image prior. They extended this method to a more general noise model.

\subsection{SR Based on Interpolation of Wavelet Domain}

Gholamreza Anbarjafari and Hasan Demirel [17] proposed a super-resolution technique based on interpolation of the high-frequency sub band images obtained by discrete wavelet transform (DWT) and the input image. They used DWT to decompose an image into different subband images. Then the highfrequency subband images and the input lowresolution image have been interpolated, followed by combining all these images to generate a new superresolved image by using inverse DWT. Figure 3 shows the block diagram of the method proposed by Gholamreza Anbarjafari and Hasan Demirel.

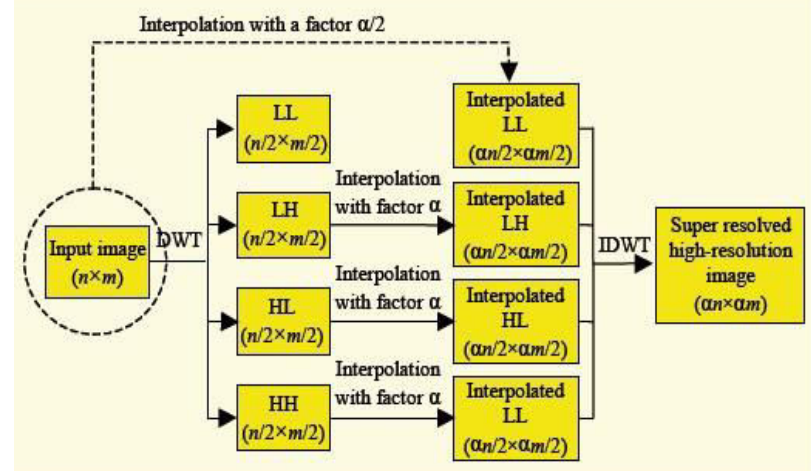

Figure 3: Block Diagram of DWT based SR

\subsection{SR by Complex Wavelet Transform}

Gholamreza Anbarjafari and Hasan Demirel [18] proposed a technique to enhance to resolution of satellite images based on interpolation of highfrequency subband images obtained by dual-tree complex wavelet transform (DT-CWT). This method uses DT-CWT to decompose an input low-resolution satellite image into different subband images and interpolates input images followed by combining all these images to generate high-resolution images by using inverse DT-CWT. Figure 4 shows the diagram of the method.

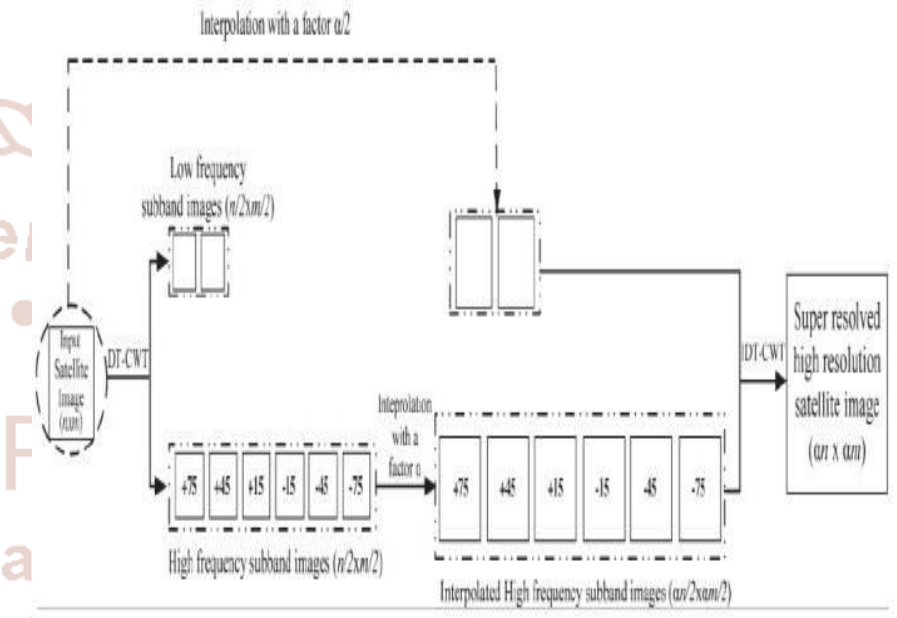

Figure 4: Block diagram of DT-CWT SR

\subsection{Image Resolution Enhancement by using Discrete and Stationary Wavelet Decomposition}

Gholamreza Anbarjafari and Hasan Demirel [19] proposed a image resolution enhancement technique based on interpolation of the high frequency subband images obtained by discrete wavelet transform (DWT) and the input image. The edges are enhanced by introducing an intermediate stage by using stationary wavelet transform (SWT). DWT is applied in order to decompose an input image into different subbands. Then the high frequency subbands as well as the input image are interpolated. The estimated high frequency subbands are being modified by using high frequency subband obtained through SWT. Then all these subbands are combined to generate a new high resolution image by using inverse DWT (IDWT).

\section{PROPOSED METHODOLOGY}

An adaptive directional image interpolation is computed by estimating sparse image mixture models in a wavelet frame. This section describes a fast orthogonal block matching pursuit implementation. The detailed process of wavelet based superresolution is, first the sub sampled image $y(n)$ for is 
decomposed with wavelet transform matrix $\psi$ whose columns are the vectors of a translation invariant wavelet frame $\psi_{d, m}$ on a single scale (the finest one). Then, it is reconstructed with a dual frame type matrix $\psi$, columns of this dual wavelet frames is $\psi_{d, m}$. The wavelet coefficients are;

$$
c(d, m)=\left\{y, \psi_{d, m}\right\}=\psi_{y}(d, m)
$$

The wavelet transform separates a low frequency image $y_{l}$ projected over the low-frequency scaling filters $\psi_{l}(d, m) \&$ a high-frequency image $y_{h}$ projected over the finest scale wavelets in three directions $\psi_{y}(h, m)$.

$$
y_{l}=\sum_{m \in G} c(l, m) \psi_{l, m}
$$$$
\& y_{h}=\sum_{d=1}^{3} c(h, m) \psi_{h, m}
$$

The low frequency image has little aliasing \& can, thus, be precisely interpolated with a cubic spline interpolator. Detailed process is;

Step 1: Computes a 1-D interpolation in the direction. We consider all lines of angle that intersect original image samples (crosses in Fig. 5) \& we compute midpoints (circles) between image samples (crosses), with a cubic spline interpolation. This operation oversamples by a factor two either the image rows, or the image columns, or the diagonals of angle. The missing coefficients are shown as squares in Fig. 4.1.

Step 2: Calculation of new samples or dots with a cubic spline interpolation along these oversampled rows, columns or diagonals. This interpolation introduces little aliasing because of the oversampling provided by the previous step. The positions of these new samples (dots) are chosen so that any missing coefficient (square) is a mid-point between two dots on a line of angle.

For every single angle, application of directional interpolator is performed for the block of wavelet components if the directional regularity factor is less in that block. Such regularization is very much helpful, when the eigen values have significantly large variation, such that discrimination between regular \& non-regular variations in the direction of angle, can be done. This is obtained by choosing rectangular blocks that are elongated in the direction of angle. Each block in the spatial neighborhood of is chosen to be identical in the three directions $1,2,3 \&$ so on. Numerical experiments are performed with 20 angles, with blocks having a width of 2 pixels \& a length between 6 \& 12 pixels depending upon their orientation. Each block, thus, includes between $36 \&$ 72 wavelet coefficients over the 1, 2, 3 directions.

An adaptive interpolation estimator by using block matching is obtained by estimating the mixing coefficients of a mixture model which minimizes the errors. The block matching technique performs the tasks of maxima finding \& then energy update i.e. find the energy of blocks, if this energy is less than threshold than eliminate all blocks.

This algorithm stops when there is no sufficiently energetic block compared to a precision threshold. The minimization is, also interpreted as an optimized approximation in orthogonal bundles computed over adapted blocks of wavelet coefficients. The adaptive wavelet interpolator is derived by taking IDWT of the resulting mixing coefficients.

\section{PROPOSED ALGORITHM}

Step 3: computes missing samples (squares) with a cubic spline linear interpolation along the direction from the previously calculated new samples (dots).

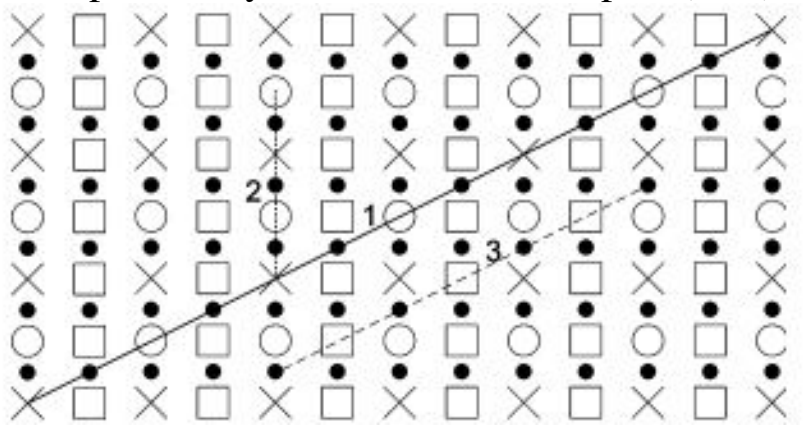

Figure 5: Directional interpolation 
International Journal of Trend in Scientific Research and Development (IJTSRD) ISSN: 2456-6470

1. Take low resolution input image.

2. This subsampled image is decomposed with wavelet transform.

3. Apply cubic spline interpolation for removal of aliasing of low frequency components. This process has following sub-steps:

(i) First computes a 1-D interpolations in the direction.

(ii) Then computes new samples by Cubic Spline interpolation.

(iii) After that missing samples are find out by again Cubic Spline interpolation.

4. For each angle, a directional interpolator is applied over a block of wavelet coefficients if the directional regularity factor is relatively small in the block.

5. Apply adaptive interpolation estimator by using block matching, for Maxima Finding \& Energy Update.

6. The adaptive wavelet interpolator is derived by taking inverse wavelet transform of the resulting mixing coefficients.

7. This will result an image having more number of pixels, compared to the input LR image, means the resultant image after application of above steps is a HR image.

8. Calculate the performance parameters like PSNR, MSE etc. and compare them with other techniques.

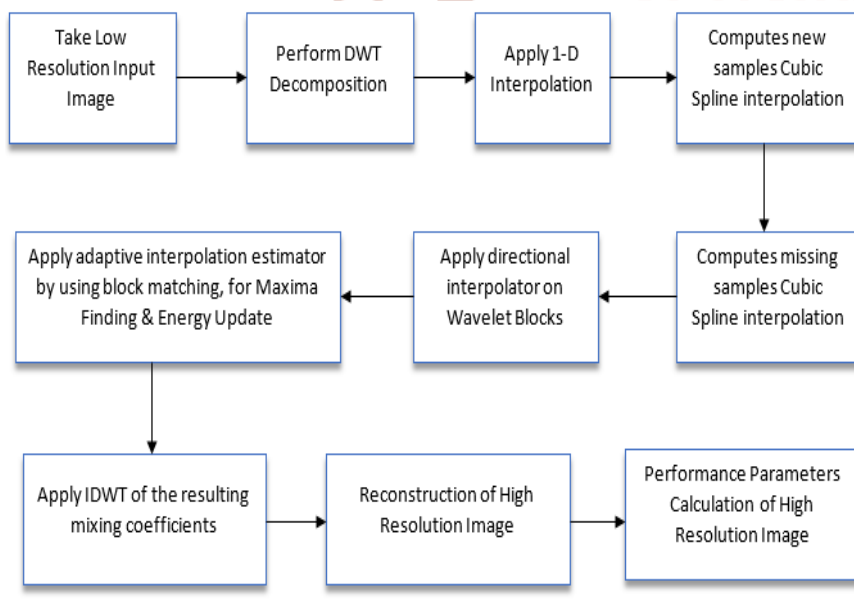

Figure 6: Proposed Wavelet based Super-Resolution Flow Chart

\section{SIMULATION RESULTS}

The proposed method is tested on various standard images Lena; Baboon \& Pepper are taken. All the input low-resolution images interpolated with factor 2 . The resolution of input image was $128 \times 128$. These input images are interpolated to the size of 512 $\times$ 512.For quantitative comparison of results peak signal to noise ratio (PSNR) \& SSIM is used.

\subsection{Performance Parameters}

The performance parameters of image are MSE, PSNR, SSIM \& many more. But, for image superresolution PSNR \& SSIM are highly used.

Peak Signal-to-Noise Ratio as a performance metric, which is measured in decibels $(\mathrm{dB})$ for 8-bit grayscale images as; $P S N R=10 \log _{10}\left(\frac{255^{2}}{M S E}\right)$

Where, the mean square error (MSE), defined as:

$$
M S E=\frac{1}{M \times N}\|f-\hat{f}\|^{2}=\frac{1}{M \times N} \sum_{*=1}^{M} \sum_{y=1}^{N}[f(x, y)-\hat{f}(x, y)]^{2}
$$

Where; $\|.\|^{2}$ is the Euclidean norm.

A larger value of PSNR normally reflects the better performance for image analysis. The PSNR \& MSE are generally used as the performance indices. But these are not so well matched to perceive visual quality of image directly. To resolve these problems, the structural similarity index (SSIM) [24] was proposed as another metric to compare images which correlates more appropriately with the human perception. SSIM basically maps two separate images into a single index in the interval $[-1,1]$, where higher values are assigned to more similar pairs of images $\mathrm{X}$ \& Y, calculated as;

$$
S S I M=\frac{\left(2 \mu_{x} \mu_{y}+c_{1}\right)\left(2 \sigma_{x y}+c_{2}\right)}{\left(\mu_{x}^{2}+\mu_{y}^{2}+c_{1}\right)\left(\sigma_{x}^{2}+\sigma_{y}^{2}+c_{2}\right)}
$$

Where; $\mu Y, \sigma X 2 \& \sigma Y 2$ are the averages \& variances of $\mathrm{X} \& \mathrm{Y}, \sigma X Y$ is the covariance between $\mathrm{X} \& \mathrm{Y}$, both $C 1 \& C 2$ are predefined constants.

\subsection{Simulation Results}

\subsubsection{TEST IMAGE LEAVES}

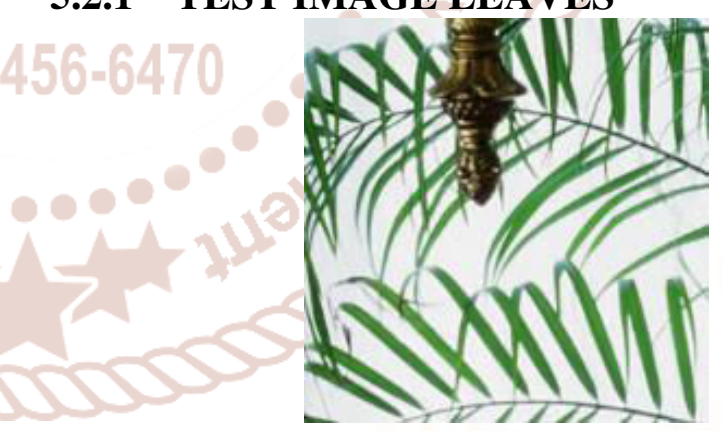

FIGURE 7: TEST IMAGE "LEAVES"
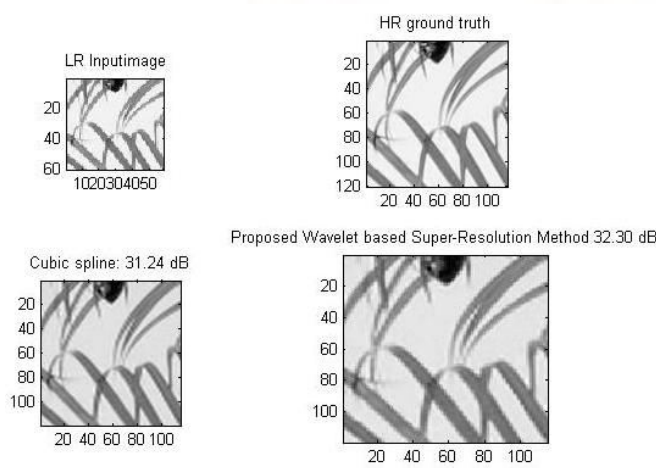

FIGURE 8: SIMULATION RESULT FOR TEST IMAGE "LEAVES" 
International Journal of Trend in Scientific Research and Development (IJTSRD) ISSN: 2456-6470

\subsubsection{TEST IMAGE HAT}

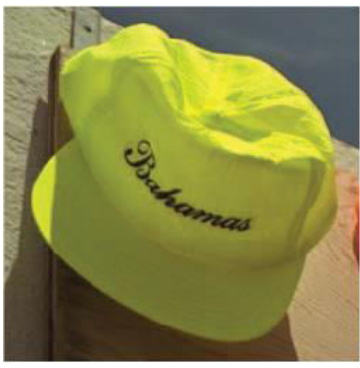

FIGURE 9: TEST IMAGE "HAT"
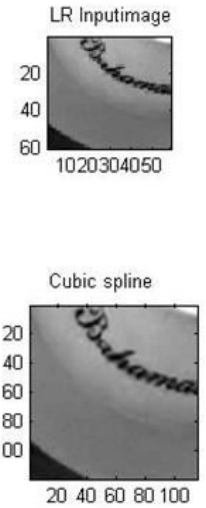

Proposed Wavelet based Super-Resolution Method $37.08 \mathrm{~dB}$

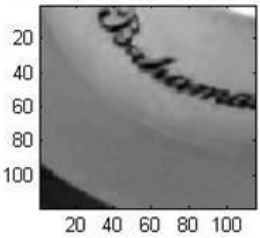

\begin{tabular}{|c|c|c|}
\hline Test Images & PSNR (dB) & SSIM \\
\hline Leaves & 32.30 & 0.9667 \\
\hline Hat & 37.08 & 0.9834 \\
\hline Mountain Lion & 41.82 & 0.9821 \\
\hline
\end{tabular}

TABLE1: SIMULATION RESULTS SUMMARY

PSNR PERFORMANCE

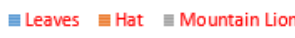

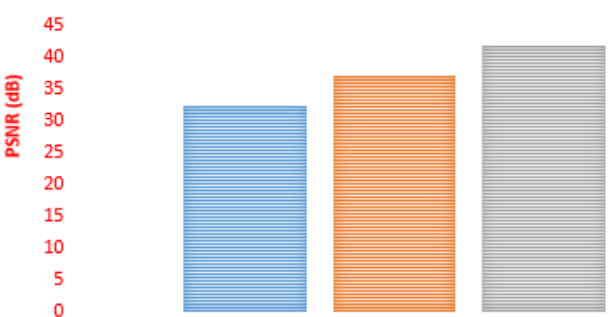

SSIM Performance

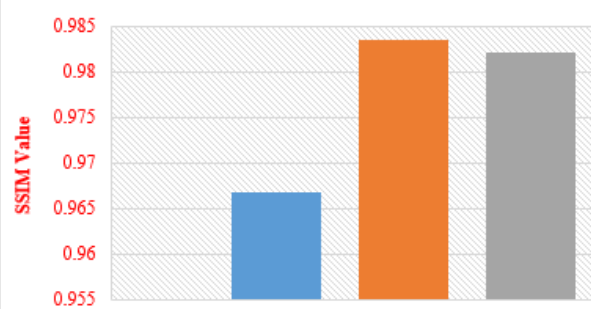

- Leaves = Hat $=$ Mountain Lion

\subsubsection{TEST IMAGE MOUNTAIN LION}

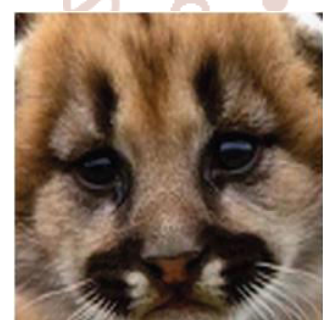

FIGURE 11: TEST IMAGE "MOUNTAIN LION"
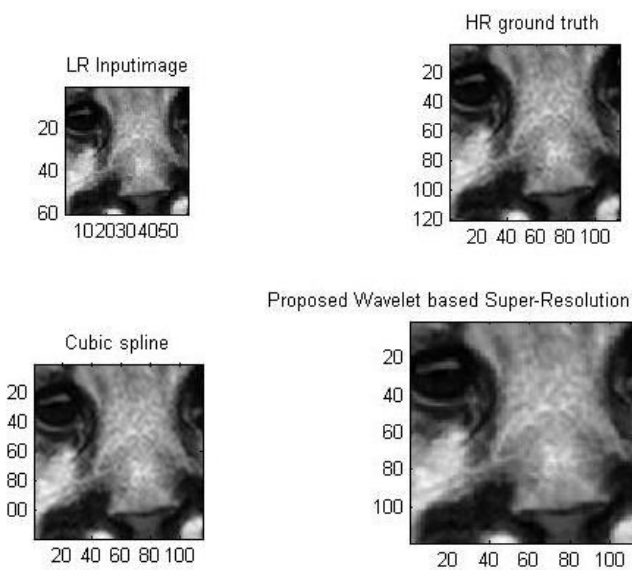

Proposed Wavelet based Super-Resolution Method $41.82 \mathrm{~dB}$

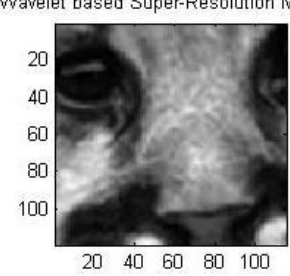

\section{FIGURE 12: SIMULATION RESULT FOR TEST IMAGE "MOUNTAIN LION"}

\subsection{SIMULATION RESULT SUMMARY}

Table 1 shows the simulation results for various test images for PSNR and SSIM values.

\subsection{RESULT COMPARISON}

The PSNR and SSIM values with proposed algorithm has been compared with [1]. Results shows our method is better than all existing techniques.

\begin{tabular}{|c|c|c|c|c|c|c|}
\hline \multirow{2}{*}{$\begin{array}{c}\text { Test Images } \\
\text { Methods }\end{array}$} & \multicolumn{2}{|c|}{ Leaves } & \multicolumn{2}{c|}{ Mountain Lion } & \multicolumn{2}{c|}{ Hat } \\
\hline SCSR & 19.21 & 0.6771 & 25.28 & 0.6388 & 26.29 & 0.79 \\
\hline CCSR & 19.24 & 0.6712 & 25.33 & 0.6424 & 26.31 & 0.7885 \\
\hline AS-CSR & 19.25 & 0.6722 & 25.34 & 0.6432 & 26.32 & 0.7894 \\
\hline TV & 24.58 & 0.8878 & 27.53 & 0.7076 & 29.19 & 0.8569 \\
\hline Softcut & 24.34 & 0.8372 & 27.96 & 0.6904 & 28.68 & 0.8389 \\
\hline SR-CNN & 24.1 & 0.8125 & 28.66 & 0.7441 & 27.82 & 0.8013 \\
\hline ASR-SL [1] & 24.6 & 0.8621 & 28.9 & 0.7588 & 29.93 & 0.8433 \\
\hline Proposed Work & $\mathbf{3 2 . 3}$ & $\mathbf{0 . 9 6 6 7}$ & $\mathbf{4 1 . 8 2}$ & $\mathbf{0 . 9 8 2 1}$ & $\mathbf{3 7 . 0 8}$ & $\mathbf{0 . 9 8 3 4}$ \\
\hline
\end{tabular}

TABLE 2: SIMULATION RESULT COMPARISON WITH [1]

\section{CONCLUSION}

The proposed wavelet based interpolation method outperforms all other existing methods for single image super resolution. The proposed method has 7.7 $\mathrm{dB}$ improvement in PSNR compared with Adaptive sparse representation and self-learning ASR-SL [1] for test image Leaves, $12.92 \mathrm{~dB}$ improvement for test image Mountain Lion \& $7.15 \mathrm{~dB}$ improvement for test image Hat compared with ASR-SL [1]. Similarly, 
$12 \%$ improvement in SSIM for test image Leaves compared with [1], 29\% improvement in SSIM for test image Mountain Lion compared with [1] \& 17\% improvement in SSIM for test image Hat compared with [1].

\section{REFERENCES}

1. Jianwei Zhao et-al, "Image super-resolution via adaptive sparse representation and self-learning", Published in IEEE IET Computer Vision, Volume: 12, Issue 5, 2018.

2. Wuzhen Shi et-al, "Single Image SuperResolution with Dilated Convolution based MultiScale Information Learning Inception Module", IEEE International Conference on Image Processing (ICIP), 2017.

3. Y. Sun, G. Gu, X. Sui, Y. Liu, and C. Yang, "Compressive Super-resolution Imaging Based on Local and Nonlocal Regularizations," IEEE Photonics Journal, Volume 8, No. 1, 2016.

4. Y. Sun, G. Gu, X. Sui, Y. Liu, and C. Yang, "Single image super-resolution using compressive sensing with a redundant dictionary," IEEE Photonics Journal, volume 7, No. 2, 2015.

5. Mayank Agrawal, Ratnakar Dash, "Image Resolution Enhancement Using Lifting Wavelet and Stationary Wavelet Transform", IEEE International Conference on Electronic Systems, Signal Processing and Computing Technologies (ICESC), 2014.

6. Mayank Agrawal, Ratnakar Dash, "Image Superresolution by Interpolating High Frequency Subbands of Image using Surface Fitting", IEEE International Conference on Signal Processing and Communication (ICSC), 2014.

7. Do Kyung Shin, Young Shik Moon, "SuperResolution Image Reconstruction Using Wavelet Based Patch and Discrete Wavelet Transform", Journal of Signal Processing Systems, Springer, Volume 81, Issue 1, pp 71-81, 2014.

8. V. Prasath R. Buvanesvari, N. Thilartham, K. Nirosha, "Image Super Resolution Reconstruction Using Wavelet Transform Method", International Journal of Innovative Technology and Exploring Engineering, Volume-3, Issue-9, 2014.

9. K. Zhang, X. Gao, D. Tao, and X. Li, "Single image super-resolution with non-local means and steering kernel regression," IEEE Trans. Image
Process., vol. 21, no. 11, pp. 4544-4556, Nov. 2012.

10. G. Yu, G. Sapiro, and S. Mallat, "Solving inverse problems with piecewise linear estimators: From Gaussian mixture models to structured sparsity," IEEE Trans. Image Process., vol. 21, no. 5, pp. $2481-2499$, May 2012.

11. W. Dong, L. Zhang, G. Shi, and X. Wu, "Image deblurring and super-resolution by adaptive sparse domain selection and adaptive regularization," IEEE Trans. Image Process., vol. 20, no. 7, pp. 1838-1857, Jul. 2011.

12. L. Zhang, L. Zhang, X. Mou, and D. Zhang, "FSIM: A feature similarity index for image quality assessment," IEEE Trans. Image Process., vol. 20, no. 8, pp. 2378-2386, Aug. 2011.

13. J. Yang, J. Wright, T. S. Huang, and Y. Ma, "Image super-resolution via sparse representation," IEEE Trans. Image Process., vol. 19, no. 11, pp. $2861-2873$, Nov. 2010.

14. X. Zhang, M. Burger, X. Bresson, and S. Osher, "Bregmanized nonlocal regularization for deconvolution and sparse reconstruction," SIAM J. Imag. Sci., vol. 3, no. 3, pp. 253-276, Jul. 2010.

15. H. Zhang, J. Yang, Y. Zhang, and T. S. Huang, "Non-local kernel regression for image and video restoration," in Proc. Eur. Conf. Comput. Vis., 2010, pp. 566-579.

16. J. Mairal, F. Bach, J. Ponce, G. Sapiro, and A. Zisserman, "Non-local sparse models for image restoration," in Proc. IEEE Conf. Comput. Vis. Pattern Recog., 2009, pp. 2272-2279.

17. M. Protter, M. Elad, H. Takeda, and P. Milanfar, "Generalizing the nonlocal-means to superresolution reconstruction," IEEE Trans. Image Process., vol. 18, no. 1, pp. 36-51, Jan. 2009.

18. P. Sen and S. Darabi, "Compressive image superresolution," in Proc. Conf. 43rd Asilomar Conf. Signals, System Computer, pp. 1235-1242, 2009

19. M. Li and T. Nguyen, "Markov random field model-based edge-directed image interpolation," IEEE Trans. Image Process., vol. 17, no. 7, pp. $1121-1128$, Jul. 2008.

20. R. Baraniuk, "Compressive sensing," IEEE Signal Process. Mag., vol. 24, no. 4, pp. 118-121, Jul. 2007. 
21. H. Takeda, S. Farsiu, and P. Milanfar, "Kernel regression for image processing and reconstruction," IEEE Trans. Image Process., vol. 16, no. 2, pp. 349-366, Feb. 2007.

22. L. Zhang and $\mathrm{X}$. Wu, "An edge-guided image interpolation algorithm via directional filtering and data fusion," IEEE Trans. Image Process., vol. 15, no. 8, pp. 2226-2238, Aug. 2006.

23. A. Buades, B. Coll, and J. M. Morel, "A review of image denoising algorithms, with a new one," Multisc. Model Simul., vol. 4, no. 2, pp. 490-530, 2005.

24. J. M. Bioucas and M. A. T. Figueiredo, "A new TwIST: Two-step iterative shrinkage/thresholding algorithms for image restoration," IEEE Trans. Image Process., vol. 16, no. 12, pp. 2992-3004, Dec. 2007.

25. Daubechies, M. Defriese, and C. Demol, "An iterative thresholding algorithm for linear inverse problems with a sparsity constraint," Commun. Pure Appl. Math. vol. 57, no. 11, pp. 1413-1457, Nov. 2004.

26. Z. Wang, A. C. Bovik, H. R. Sheikh, and E. P. Simoncelli, "Image quality assessment: From error measurement to structural similarity," IEEE Trans. Image Process., vol. 13, no. 4, pp. 600612, Apr. 2004.

27. S. C. Park, M. K. Park, and M. G. Kang, "Superresolution image reconstruction: A technical overview," IEEE Signal Process. Mag., vol. 20, no. 3, pp. 21 -36, May 2003.

28. X. Li and M. T. Orchard, "New edge-directed interpolation," IEEE Trans. Image Process., vol. 10, no. 10 , pp. 1521 - 1527, Oct. 2001.

29. H. Knutsson and C. F. Westin, "Normalized and differential convolution," in Proceeding of IEEE Conference on Computer Visual Pattern Recognition, pp.515-523, 1993. 